\title{
Avoiding chemotherapy related late effects in children with curable tumours
}

\author{
C Ross Pinkerton
}

A number of articles have highlighted the dilemma facing those involved with the design of treatment programmes for patients with curable tumours, in whom long term survival necessitates the avoidance, if possible, of late sequelae. $^{2}$ Nowhere is this problem more apparent than in the management of paediatric cancer. With almost $60 \%$ of children who have been treated for cancer in the past decade achieving long term survival and probable cure, it is becoming increasingly clear that a variety of problems are being created as a direct consequence of curative treatment. Many paediatric subspecialists other than oncologists, such as endocrinologists, cardiologists, respirologists, and neurologists are involved in the joint long term follow up of these children into adulthood. The problem of avoiding mutilating surgery has largely been achieved by the use of preoperative chemotherapy. Similarly, extended field high dose radiotherapy is now rarely used in paediatric practice. These improvements that avoid late sequelae are, however, dependent on the effective use of chemotherapy to achieve maximum response and reduce or obviate the need for further treatment directed towards local tumour control.

In an attempt to reduce chemotherapy related late sequelae there is at present considerable debate concerning which drugs could be dropped or replaced by less toxic agents. Virtually all chemotherapeutic agents are non-specific cell poisons; their effectiveness often depending on a narrow therapeutic ratio (that is, tumour cell kill to normal tissue toxicity). Up until recently the classic 'bogymen' of chemotherapy were the alkylating agents. The use of regimens such as mustine, vincristine, procarbazine, prednisolone (MOPP) or chlorambucil, vincristine, procarbazine, prednisolone (ChlVPP) in childhood Hodgkin's disease is undesirable if effective alternatives exist because of the inevitable sterilising effect in males and the possible risk of second tumours and leukaemia. There is little doubt about the sterilising effect in boys after standard chemotherapy for Hodgkin's disease, although girls are generally spared this adverse effect.

Data for cyclophosphamide alone are less clear, but it seems likely that a third to a half of boys will be sterilised after conventional 12-24 months regimens for various childhood solid tumours. ${ }^{3}$ It has been suggested that a total cumulative dose of up to around $4 \mathrm{~g} / \mathrm{m}^{2}$ of cyclophosphamide can be given without sterilisation. ${ }^{4-6}$ With regimens containing a small number of courses using high doses of cyclophosphamide, for example in non-Hodgkin's lymphoma, where up to $3 \mathrm{~g} / \mathrm{m}^{2}$ is given per course, ${ }^{78}$ the sterilising effect may be less than when the same total dose is given over several months. This remains to be clarified, however, in prospective studies. Ifosfamide, an analogue of cyclophosphamide, which appears to be less myelosuppressive with comparable antitumour alkylating activity, is now in widespread use for Ewing's sarcoma, rhabdomyosarcoma, and some protocols for leukaemia/lymphoma. Unfortunately the lack of myelosuppression has been offset by a disturbing incidence of renal tubulopathy. The precise incidence of significant dysfunction remains unclear pending adequate studies of unselected patients, but there is little doubt that a Fanconi-like syndrome may occur particularly in the smaller child who has received high doses of ifosfamide. The sterilising effect of four to six courses of $6-9 \mathrm{~g} / \mathrm{m}^{2}$ ifosfamide is unclear but seems likely.

The incidence of second cancers in children ranges from $1-4 \%$ and is dependent on the nature of prior chemotherapy and the use of radiotherapy. ${ }^{9}$ In adults an incidence of up to $18 \%$ at 15 years has been reported in those with Hodgkin's disease who have received alkylating agents and radiotherapy. ${ }^{10}$ In one study of childhood Hodgkin's disease using an alternating MOPP/doxorubicin bleomycin, vinblastine, dacarbazine (ABVD) regimen where the median follow up was only 35 months three out of 62 patients developed second malignancies. The latter attempt to reduce the late effects of a MOPP based regimen could in fact result in 'the worst of both worlds', namely sterilisation, second tumours, anthracycline related cardiotoxicity, and bleomycin related lung toxicity. ${ }^{11}$ With a conservative radiotherapy policy risks are lower and only three second malignancies have occurred in 232 children mostly receiving ChlVPP alone on the current United Kingdom Children's Cancer Study Group (UKCCSG) regimen. High incidences have been reported in children with Ewing's sarcoma treated with cyclophosphamide based regimens and high dose radiotherapy ${ }^{12}$ and in children with nonHodgkin's lymphoma receiving cyclophosphamide and chloroethylcyclohexyl nitrosourea (CCNU) regimens. ${ }^{13}$

In solid tumours such as rhabdomyosarcoma and Wilms' tumour a series of prospective randomised studies have evaluated the need to include cyclophosphamide. The American Intergroup Rhabdomyosarcoma Study group has demonstrated clearly that this drug is not necessary to cure patients with completely resected rhabdomyosarcoma or in those with microscopic residual disease provided they are
Downs Road, Surrey SM2 5PT Correspondence to: Dr Pinkerton. 
irradiated. ${ }^{14} 15$ In the case of Wilms' tumour, cyclophosphamide is not required but with more extensive tumour the omission of this agent must be off set by the retention of an anthracycline; this is a debatable compromise. ${ }^{16}$

In non-Hodgkin's lymphoma the overall cure rates now exceeds $80 \%$ and the necessity for including cyclophosphamide is currently being evaluated by the UKCCSG. In the UKCCSG 9001 regimen children with localised (Murphy stage 1 and 2) disease receive a non-alkylating agent regimen that does include an anthracycline. The French paediatric oncology group has reduced the duration of chemotherapy to two courses of cyclophosphamide, doxorubicin, vincristine, prednisolone (CHOP) for patients with stage 1 and 2 disease in the hope that the total dose of alkylating agent and anthracycline will be sufficiently low to avoid late effects. The American CCSG has opted to omit the anthracycline using the cyclophosphamide based cyclophosphamide, vincristine, methotrexate, prednisolone (COMP) regimen. Which of these options is preferable will only become apparent with further follow up in each study when information regarding sterility and cardiotoxicity is available. Attempts to avoid the alkylating agents in Hodgkin's disease include the ABVD and vincristine, etoposide, epirubicin, and prednisolone regimen. ABVD has the disadvantage of potential lung toxicity from bleomycin and doxorubicin cardiotoxicity. In VEEP chlorambucil and procarbazine are replaced by etoposide and epirubicin, which are nonsterilising, and it was hoped that the late cardiotoxicity of epirubicin would be less than with doxorubicin. ${ }^{17}$ The ABVD regimen has been shown in both adults and children to be equally effective to MOPP or ChlVPP. With VEEP, however, although response rates in adults have been comparable with that seen with the ChIVPP regimen, this has not been the case in a pilot childhood study where the incidence of initial refractory disease has been worrying. ${ }^{17}$ The strategy of using an etoposide based regimen relies on a high salvage rate, in the event of failure, using ChIVPP and radiotherapy. In this way even if there is a higher relapse rate using the VEEP regimen the overal cure rate should be comparable but the majority of boys will not be sterilised and it is hoped that second tumours will be fewer. This approach remains controversial as it involves potentially suboptimal treatment in order to avoid sterility, which should be effective in most, but accepts that the minority who relapse will end up having even more intensive treatment to achieve cure. This therefore raises the issue of how important is fertility?

Recent evidence on late effects of anthracyclines is one of the most worrying aspects of paediatric chemotherapy. ${ }^{12}{ }^{18} \mathrm{Up}$ until recently it was felt that a cumulative dose of up to 350 $\mathrm{mg} / \mathrm{m}^{2}$ of doxorubicin was 'safe' and such doses may be included in regimens for acute lymphoblastic leukaemia, acute myeloid leukaemia, and Ewing's sarcoma. This now appears to have been an underestimate. For a number of years there have been anecdotal reports of children treated for solid tumours developing cardiac insufficiency during pregnancy or after severe exercise, but these patients had usually received high cumulative doses. The demonstration by sophisticated echocardiography of myocardial impairment in children with leukaemia who have received considerably less than the conventional safe dose suggests that this may not in fact exist. Unfortunately at present no reliable method of monitoring cardiac function during treatment allows cessation of anthracyclines before significant damage occurs. Endomyocardial biopsy is the only definitive way to detect early anthracycline damage. For obvious reasons data are very limited in children and interpretation of any changes seen with regard to functional prognosis would be difficult. Electrocardiography or conventional echocardiography are only able to detect established damage. Recent improvement with $M$ mode and colour flow Doppler may improve sensitivity but standardisation in methodology is required for multicentre use.

Strategies to try and reduce the cardiotoxicity of anthracyclines include prolonged continuous infusion schedules or the use of new analogues such as epirubicin, idarubicin, the substitution of doxorubicin with mitozantrone, or the use of cardioprotector drugs. In lymphoma or leukaemia where there are many potential active agents, the necessity for anthracyclines must be questioned. In the case of actue lymphoblastic leukaemia, there is little randomised study evidence supporting the need for this drug, particularly in the context of alternative combinations such as high dose cytarabine, etoposide, and high dose methotrexate. This issue needs to be urgently addressed in large national multicentre studies.

With solid tumours where non-cardiotoxic, non-sterilising agents such as cisplatin have activity, reluctance to use this drug because of its nephrotoxicity and ototoxicity may have been overcome by the introduction of its analogue carboplatin. There is, however, the trade off of a different toxicity, myelosuppression, that may result in dose reduction in combination treatment and thus have an adverse effect on efficacy. Moreover, the comparable activity of carboplatin needs to be clearly demonstrated in each of the relevant solid tumours. ${ }^{19}$

What other group of drugs should be considered to replace the major offending agents? Unfortunately most of the antimetabolites, such as methotrexate and cytarabine, which have few late sequelae have limited activity in solid tumours. Although well tolerated, the use of bleomycin in lymphomas or germ cell tumours is to be avoided because of the late effects on pulmonary function. It is likely that this drug will be omitted completely from most paediatric schedules in the near future once it has been demonstrated that its inclusion in the PVB (cisplatin, vinblastine, bleomycin) or BEP (bleomycin, etoposide, cisplatin) germ cell tumour regimen in adults is not necessary, provided sufficient doses of the other drugs are given.

The epipodophyllotoxins, etoposide or teniposide, have achieved popularity in recent years with demonstration of activity in leukae- 
mia, lymphoma, Wilms' tumour, rhabdomyosarcoma, and neuroblastoma. Continuing studies are evaluating the precise role of etoposide but its apparent lack of late effects made it a very attractive agent to replace alkylating agents and anthracyclines in many tumours. These hopes have been somewhat dampened recently by reports describing an apparent increase in secondary leukaemias in patients treated with teniposide/etoposide based regimens for both solid tumours and leukaemia. In a number of these reports etoposide has been given in combination with alkylating agents, making it difficult to be sure about its precise contribution. In small cell lung cancer and malignant germ cell tumours etoposide or teniposide do appear to be implicated. ${ }^{20}{ }^{21}$ In these reports the dose of etoposide has been higher than that currently used in most paediatric protocols. There have to date been very few reports of secondary leukaemias in children given etoposide or teniposide for solid tumours. ${ }^{22}$ In one frequently quoted study where a high incidence of secondary acute myeloid leukaemia was observed after treatment for non-Hodgkin's lymphoma, in addition to teniposide the well known carcinogen CCNU was included in the protocol. ${ }^{13}$ There has, however, been some more convincing evidence in childhood acute lymphoblastic leukaemia where the schedule of etoposide and teniposide appear to be important. Children receiving frequent administration of these agents, that is once or twice a week, appeared to be at significant risk. ${ }^{23}$ Again the total doses were considerably greater than those used in solid tumours with a median total dose of $6 / \mathrm{m}^{2}$ in those affected, with only one case occurring with a total dose of $<3 \mathrm{~g} / \mathrm{m}^{2}$. The high percentage of cases with involvement of chromosome $11 \mathrm{q} 23$, which has been observed after in vitro treatment of cell lines with etoposide,${ }^{24}$ is further supportive evidence of its 'leukaemic' effect when given at high doses.

In patients with acute lymphoblastic leukaemia there was no increased risk for children given etoposide on an alternate weekly basis, and where the drug is given once every three weeks as in most solid tumour regimens it is likely to be safe. There is, however, evidence that the more frequent scheduling of this agent may be more effective. ${ }^{25} 26$ It is probably wise to limit such regimens to those with high risk disease or after relapse where improved efficacy could outbalance the small but significant risk of transforming mutations in normal myeloid progenitor cells.

In conclusion, it is clear that we cannot expect effective curative chemotherapy to be devoid of all late sequelae. There are, however, children with tumours where at present the cure rate exceeds $70 \%$, for example, acute lymphoblastic leukaemia, Wilms' tumour, malignant germ cell tumours, non-Hodgkin's lymphoma, Hodgkin's disease, and localised soft tissue sarcomas. For these patients protocol design will rest upon whether one accepts infertility rather than the risk of late cardiomyopathy or if soft tissue and bone hypoplasia due to localised radiotherapy is preferable to a small risk of chemotherapy related second leukaemias. This is a topic of intense debate among paediatric oncologists and many of the points raised require clarification. Because of the comparatively small number of patients involved and the high cure rates seen, prospective randomised studies evaluating the effect of omitting potentially toxic agents or the alternative late effects of newer drugs need to be done on a large multicentre, probably international, basis. The International Society for Paediatric Oncology, SIOP, is one forum where this could be done and should be encouraged to start in the near future. Empirical changes without adequate prospective evaluation may lead to replacing one toxicity by another unexpected one-out of the frying pan, into the fire.

1 Yeung ST, Yoong C, Spink J, Galbraith A, Smith PJ. Functional myocardial impairment in children treated with anthracyclines for cancer. Lancet 1991;337:816-8.

2 Lipshultz SE, Colan SD, Gelber RD, et al. Late cardiac effects of doxorubicin therapy for acute lymphoblastic leukemia in childhood. $N$ Engl f Med 1991;324:808-15.

3 Byrne J, Mulvihill JJ, Myers MH, et al. Effects of treatment on fertility in long-term survivors of childhood or adolescent on fertility in long-term survivors of child
cancer. $N$ Engl F Med 1987;317:1315-21.

4 Aubier F, Flamant F, Brauner R, et al. Male gonadal function after chemotherapy for solid tumors in childhood. $\mathcal{F}$ Clin Oncol 1989;7:304-9.

5 Kreuser ED, Hetzel WD, Heit W, et al. Reproductive and endocrine gonadal functions in adults following multidrug chemotherapy for acute lymphoblastic or undifferentiated leukaemia. F Clin Oncol 1988;6:588-95.

6 Meistrich ML, Chawla SP, Da Cunha MF, et al. Recovery of sperm production after chemotherapy for osteosarcoma. Cancer 1989;63:2115-23.

7 Patte C, Philip T, Rodary C, et al. Improved survival rate in children with stage III and IV B cell non-Hodgkin's lymphoma and leukemia using multi-agent chemotherapy: resuls of a study of 114 children from Oncology Society. F Clin Oncol 1986;4:1219-26.

8 Hann IM, Eden OB, Barnes J, Pinkerton CR. 'MACHO' chemotherapy for stage IV B cell lymphoma and B cell chemotherapy for stage IV B cell lymphoma and B cell 1990;76:359-64.

9 de Vathaire F, Schweisguth O, Rodary C, et al. Long-term risk of second malignant neoplasm after a cancer in risk of second malignant neoplasm
childhood. Brf Cancer 1989;59:448-52.

10 Kushner BH, Zauber A, Tan CTC. Second malignancies after childhood Hodgkin's disease. The Memorial SloanKettering Cancer Center experience. Cancer 1988;62: $1364-70$.

11 Weiner MA, Leventhal BG, Marcus R, et al. Intensive chemotherapy and low-dose radiotherapy for the treatment of advanced-stage Hodgkin's disease in pediatric patients: a pediatric oncology group study. F Clin Oncol 1991;9: 1591-8.

12 Tucker MA, D’Angio GJ, Boice JD, et al. Bone sarcomas linked to radiotherapy and chemotherapy in children. NEngl F Med 1987;317:588-93.

13 Ingram L, Mott MG, Mann JR, et al. Second malignancies in children treated for non-Hodgkin's lymphoma and T-cell leukaemia with the UKCCSG regimens. Br 7 Cancer 1987; leukaemia

14 Maurer HM, Beltangady M, Gehan EA, et al. The Intergroup Rhabdomyosarcoma Study-1. A final report. Cancer 1988; 61:209-20.

15 Crist WM, Garnsey L, Beltangady MS, et al. Prognosis in children with rhabdomyosarcoma: A report of the Intergroup Rhabdomyosarcoma Studies I and II. $\mathcal{f}$ Clin Oncol 1990;3:443-52.

16 D'Angio GJ, Evans A, Breslow N, et al. The treatment of Wilms' tumor: results of the Second National Wilms' Tumor Study. Cancer 1981;47:2302-11.

17 O'Brien MER, Pinkerton CR, Kingston J, et al. 'VEEP' in children with Hodgkin's disease - a regimen designed to decrease late sequelae. Br f Cancer 1992;65:756-60.

8 Doroshow JH. Doxorubicin-induced cardiac toxicity. N Engl f Med 1991;324:843-5.

19 Pinkerton CR, Lewis IJ, Pearson ADJ, Stevens MCG, Barnes J. Carboplatin or cisplatin? Lancet 1989;ii:161.

20 Whitlock JA, Greer JP, Lukens JN. Epipodophyllotoxin related leukemia. Identification of a new subset of secondary

21 Pedersen-Bjergaard J, Daugaard G, Hansen SW et al. Increased risk of myelodysplasia and leukaemia after Increased risk of myelodysplasia and leukaemia after
etoposide, cisplatin, and bleomycin for germ-cell tumours.

22 Pui C-H, Hancock ML, Raimondi SC, et al. Myeloid neoplasia in children treated for solid tumours. Lancet 1990;

23 Pui C-H, Ribeiro RC, Hancock ML, et al. Acute myeloid 
leukemia in children treated with epipodophyllotoxins for acute lymphoblastic leukemia. $N$ Engl f Med 1991;325: 1682-7.

24 Maraschin J, Dutrillaux B, Aurias A. Chromosome aberrations induced by etoposide (VP-16) are not random. Int $\mathcal{F}$ Cancer 1990;46:808-12.
25 Clark PI, Joel SP, Slevin ML. A pharmacokinetic hypothesis for the clinical efficacy of etoposide in small cell lung cancer. Brf Cancer 1989;60:458.

26 Hainsworth JD, Johnson DH, Frazier SR, et al. Chronic daily administration of oral etoposide-a phase I trial. f Clin Oncol 1989;7:396-401.

\section{Dyslexia}

It has always seemed to me that some people make very heavy weather of dyslexia. 'Do you believe in it?' they say, as if it were a cross between astrology, water divining, and Rosicrucianism. Of course I believe in it. The fact that some people of normal or high intelligence have inordinate difficulty with reading is a matter of experience as easily verifiable as the fact that some people have eyes of different colour. Ah, well if it exists, is it a disease? The New England Fournal of Medicine of 16 January 1992 includes a leading article with that title, 'Dyslexia-is it a disease?' We could get into deep water by pursuing the question of what constitutes a disease but in the context of this article the point at issue is whether children who have reading skills which are inappropriately low for their intelligence represent simply the lower end of a normal distribution curve or whether they are a separate group with distinct pathophysiology.

In the same issue of the New England Fournal of Medicine there is a paper from Yale (Sally E Shaywitz and colleagues, 1992;326: 145-50) which attempts to answer this question. The Connecticut Longitudinal Study is a follow up study of 414 children who began their schooling in 1983. They had intelligence tests performed in their first, third, and fifth school years and annual tests of reading and mathematical proficiency in their first six years at school. When reading scores were compared with full scale IQs it was apparent firstly that as expected, there is in general a fairly close correlation between intelligence and reading ability. Secondly, the distribution of reading scores for IQ seemed normal and these workers were unable to detect a 'hump' at the lower end of the scale. They conclude that there is a smooth normal distribution curve for reading ability in relation to intelligence and that children with dyslexia represent the lower end of this normal distribution, the cut off between normal and abnormal being arbitrarily defined. This does not exclude a small number of children having a pathological cause. They estimate that if the prevalence of pathological dyslexia were $5 \%$ their study would have a $90 \%$ chance of detecting a hump but at a prevalence of 0.5 to $1 \%$ their chance of showing a hump would be less than $20 \%$. So these data suggest that either there is no pathological dyslexia or its prevalence is less than it has been thought to be.

Dyslexia due to a specific defect of neuroanatomy or neurophysiology might be expected to be fairly constant but in this study there was considerable variation with time. Defining dyslexia as a reading score more than $1.5 \mathrm{SDs}$ below that expected for intelligence there was considerable movement from year to year in and out of the dyslexic group. Thus, of children defined as dyslexic in the first school year only $28 \%$ were still so defined in the third year and of those dyslexic in the third year $47 \%$ were still dyslexic in the fifth year. Equally, some children not dyslexic in the earlier school year became so later. A considerable number of children had reading scores well in advance of their intelligence. Were they superlexic? Clearly, if reading ability for IQ is normally distributed then dyslexia and superlexia should be equally prevalent. I've never heard of superlexia. Could it give rise to any problems?

What are the implications? There can be no doubt that some children underachieve as regards reading and need help. There may be a small hard core of children with stable dyslexia who need constant help but other children's reading achievement may fluctuate and they may need help at different times in their school careers. Reading disability, it seems, cannot be diagnosed once and for all in the early school years. 